\title{
Proving Ground Confluence and Inductive Validity in Constructor Based Equational Specifications
}

\author{
Klaus Becker \\ Fachbereich Informatik, Universität Kaiserslautern \\ 6750 Kaiserslautern, Germany \\ email: kIbecker@informatik. uni-kl.de
}

\begin{abstract}
We study ground confluence and inductive validity in equational specifications that are interpreted in a constructor based way. By defining semantics of the specification in an appropriate way, we are able to transfer the proof-byconsistency concepts from unstructured specifications to constructor based specifications, where the unstructured specifications with their usual semantics are included as a special case. We further show that the proof-by-consistency concepts not only apply to inductive theorem proving, but can as well be employed to prove ground confluence of rewrite systems. So we present a refutationally complete prover for ground confluence and inductive validity that applies to unstructured as well as constructor based equational specifications.
\end{abstract}

\section{Introduction}

Equational specifications can be considered as the programs of an applicative programming language with rewriting being its computation mechanism. Semantics is assigned to such specification programs using canonical term algebras. Usually all ground terms of the given signature contribute to the construction of the carrier set. Sometimes however it is convenient to introduce so-called constructors in order to capture the intuition of the specifier. In that case the operations given by the signature are split into two groups: those which are used to construct the domain of computation - the constructors - and those which do not contribute to this construction, but which are to be (possibly partially) defined over the domain of interest - the defined operators.

To consider an example, let 0 ("zero") and $s$ ("successor") be the constructor symbols that introduce the natural numbers. Then the following equations $(R 1) \ldots(R 4)$ define addition and subtraction over the natural numbers. Note that subtraction is not a totally defined operation with respect to the constructors, as there is no constructor term that is equivalent to (e.g.) $0-s(0)$.

$(R 1) \quad x+0=x$
$(R 2) x+s(y)=s(x+y)$

(R3) $x-0=x$

(R4) $s(x)-s(y)=x-y$ 
When choosing this kind of predefined split of operators, it is reasonable to interprete those equations that define non-constructor symbols in a constructor restricted way: variables occuring in these equations are to be instantiated only by constructor terms. We call the result of such an interpretation of the specification, which is in the spirit of Kapur\&Musser [KaMu86, KaMu87], "constructor semantics". In order to model the variable restrictions we use a suitable order-sorted interpretation of the syntactical objects (see also [SNGM89]).

When specifying with equations, ground confluence and inductive validity are of special interest. Ground confluence can be considered as a kind of correctness property of the specification, whenever the equations of the specification are used as rewrite rules. The notion of inductive validity is used to describe those equations that are valid in the standard model of the specification.

Whereas little work has been done on proving ground confluence (see [Pl85, Fr86, Go87]), several methods have been developed to prove inductive validity in various contexts (see e.g. [Bu69, BoMo79, Mu80, HuHu82, KaMu87, Ba88, KoRu90, Ly92]).

The constructor approach we are concerned with is treated in [KaMu86, KaMu87] using the so-called proof-by-consistency method. Problems usually occur in this approach if partially defined functions are present. Take for instance in the example above the equation $(x-y)+y=x$, where the left hand side $(x-y)+y$ is not totally defined wrt. the constructors. Of course, the question, whether this equation is an inductive theorem or not, depends on how the notion of inductive validity is defined.

Using our definition of semantics we are given a simple and canonical way to introduce inductive validity, causing no particular problems when partially defined functions are present. An equation is said to be inductively valid, if it is valid in the standard (constructor-based) model of the specification. We thus propose a quite simple solution to the problem mentioned in [KNZ91].

Our approach to treat ground confluence is based on the observation (which is also stated in [ $\mathrm{Pa} 92]$ ), that the methods to prove inductive validity can as well be employed to prove ground confluence. Our method thus totally differs from the existing ones.

To summarize, we develop a theorem prover for inductive validity and ground confluence which is based on constructor semantics. This prover is designed along the lines of [Ba88], thus leading to a refutationally complete method. That means, if the given set of hypotheses is not inductively valid, or if the given rewrite system is not ground confluent, then this fact will be indicated after a finite number of steps. Finally note that our approach is designed in such a way that it contains the case of unstruclured specifications with their usual semantics as a special case, as one always can decide to consider all symbols as constructors.

The paper is organized as follows: Section 2 introduces constructor based specifications, section 3 constructor semantics and some basic notions. Section 4 analyses the key notion of ground reducibility in the constructor based context. In section 5 we describe the prover from an abstract point of view. The specific inference rules are developed in section 6. Finally, in the last section we put together previous results and illustrate them with some examples.

We assume that the reader is familiar with the basic concepts of term rewriting (see [AvMa90, DeJo90]) and mathematical logic. Notions and notations not defined here are standard. Proofs have to be omitted for lack of space. 


\section{Constructor based specifications}

A specification $(\Sigma, \mathcal{R})$ is given by a signature $\Sigma$ and a set $\mathcal{R}$ of equations. These equations will be interpreted as rewrite rules throughout the paper. Following the lines of Kapur \& Musser [KaMu86, KaMu87] we fix the terms that are to be considered as constructors in advance. Technically, we assume that $\Sigma$ is a signature enrichment $\Sigma_{0}+\Sigma_{1}$ and that $\mathcal{R}$ is a rule enrichment $\mathcal{R}_{0}+\mathcal{R}_{1}$ compatible with the signature enrichment (see below). . $\Sigma_{0}$ introduces the sorts and basic constructor operations and $\mathcal{R}_{0}$ defines the relations among the basic objects. $\Sigma_{1}$ introduces new function symbols that are defined by $\mathcal{R}_{1}$ wrt. the constructor domain. The latter means that the variables in $\mathcal{R}_{1}$ are to be instantiated only by constructor terms.

In order to model this kind of variable restriction we use an order-sorted interpretation of $\Sigma$ (see also [SNGM89]). This approach enables us to model partial functions in a canonical way.

A (hierarchical) signature $\Sigma=(S, F, D)$ consists of a set $S$ of sort symbols, a set $F$ of function symbols and a set $D$ of function declarations $f: s_{1}, \ldots, s_{n} \rightarrow s$ $\left(f \in F ; s_{i}, s \in S\right)$ and subsort declarations $s_{1} \triangleleft s_{2}\left(s_{1}, s_{2} \in S\right)$, where $\triangleleft$ denotes the ordering relation between the sorts.

A signature $\Sigma=(S, F, D)$ is said to be flat iff $D$ contains no subsort declarations, and for any $f \in F$ there exists exactly one function declaration in $D$.

A functional signature enrichment $\Sigma_{0}+\Sigma_{1}$ consists of a signature $\Sigma_{0}=\left(S_{0}, F_{0}, D_{0}\right)$ and a triple $\Sigma_{1}=\left(\emptyset, F_{1}, D_{1}\right)$ with $F_{0} \cap F_{1}=\emptyset$ such that $\left(S_{0}, F_{1}, D_{1}\right)$ is a signature too. Note that there are introduced no new sorts by $\Sigma_{1}$.

We assume in the sequel that $\Sigma=\Sigma_{0}+\Sigma_{1}$ is a given flat functional signature enrichment. Further we assume that $V=\bigcup_{s \in S_{0}} V_{s}$ is the union of disjoint infinitary sets $V_{s}$ of variables for the sorts introduced by $\Sigma_{0}$.

Next we construct the (hierarchical) constructor signature $\Sigma^{\wedge}=\Sigma_{0}^{\wedge}+\Sigma_{1}^{\wedge}$ induced by the (flat) functional signature enrichment $\Sigma=\Sigma_{0}+\Sigma_{1}$. Let $F_{0}^{(\geq 1)}$ be the set of function symbols of $F_{0}$ that have an arity greater or equal than 1 . Then let

- $\Sigma_{0}^{\wedge}=\left(S_{0} \cup S_{0}^{\wedge}, F_{0}, D_{0} \cup D_{0}^{\wedge} \cup D_{\text {sort }}\right)$, where

$$
\begin{aligned}
& S_{0}^{\wedge}=\left\{s^{\wedge} \mid s \in S_{0}\right\} \\
& D_{0}^{\wedge}=\left\{f: s_{1}^{\wedge}, \ldots, s_{n}^{\wedge} \rightarrow s^{\wedge} \mid f \in F_{0}^{(\geq 1)}, f: s_{1}, \ldots, s_{n} \rightarrow s \in D_{0}\right\} \\
& D_{\text {sort }}=\left\{s \triangleleft s^{\wedge} \mid s \in S_{0}\right\},
\end{aligned}
$$

- $\Sigma_{1}^{\wedge}=\left(\emptyset, F_{1}, D_{1}^{\wedge}\right)$, where

$$
D_{1}^{\wedge}=\left\{f: s_{1}^{\wedge}, \ldots, s_{n}^{\wedge} \rightarrow s^{\wedge} \mid f \in F_{1}, f: s_{1}, \ldots, s_{n} \rightarrow s \in D_{1}\right\} .
$$

Now every flat $\Sigma$-term can be regarded as a hierarchical $\Sigma^{\wedge}$-term and vice versa. In the sequel we interprete all terms over the hierarchical signature $\Sigma^{\wedge}$. As a consequence, the range of the variables that are introduced for the sorts in $S_{0}$ is restricted. Note that no variables are introduced for the new sorts in $S_{0}^{\wedge}$.

Let $\operatorname{TERM}\left(\Sigma^{\wedge}, V\right)$ denote the set of $\Sigma^{\wedge}$-terms, $\operatorname{TER} M_{0}\left(\Sigma^{\wedge}, V\right)$ the set of terms of a sort in $S_{0}$ - the so-called base terms - and $\operatorname{TERM}^{\wedge}\left(\Sigma^{\wedge}, V\right)$ the terms of a sort in $S_{0}^{\wedge}$ - the mixed terms. 
A $\Sigma^{\wedge}$-compatible rule enrichment $\mathcal{R}=\mathcal{R}_{0}+\mathcal{R}_{1}$ is the union of the sets of rules $\mathcal{R}_{0}$ and $\mathcal{R}_{1}$ (satisfying the usual variable condition $V A R(v) \subseteq V A R(u)$ for $u=v \in \mathcal{R}$ ) such that (i) $\mathcal{R}_{0}$ is a set of rules over $\Sigma_{0}$ and (ii) $\mathcal{R}_{1}$ is a set of rules over $\Sigma^{\wedge}$, where the left hand side of every rule in $\mathcal{R}_{1}$ contains a new symbol of $F_{1}$ (or is an element of $\left.\operatorname{TER}^{\wedge}\left(\Sigma^{\wedge}, V\right)\right)$.

Finally, a constructor based specification $\left(\Sigma^{\wedge}, \mathcal{R}\right)$ consists of a constructor signature $\Sigma^{\wedge}=\Sigma_{0}^{\wedge}+\Sigma_{1}^{\wedge}$ induced by a flat functional signature enrichment $\Sigma=\Sigma_{0}+\Sigma_{1}$ and a $\Sigma^{\wedge}$-compatible rule enrichment $\mathcal{R}=\mathcal{R}_{0}+\mathcal{R}_{1}$.

We finish this section with some remarks about the kind of specifications just introduced. First, the order-sorted approach is well-suited to capture a "constructor based intuition" in a rather simple and natural way. Special technical problems do not occur, as the sort hierarchy is such simple that all the basic notions to be defined below remain decidable. In particular, the rules introduced by the specification are always sort-decreasing, thus leading to a well-behaved rewrite relation.

There is one point that always should be kept in mind: Variables do only exist for the base sorts. Consequently, to be sort-compatible, all the substitutions used throughout the paper (including matching substitutions and unifiers) have to instantiate variables by base terms. In order to get rid of this restriction, we introduce what we call quasi-substitutions (see section 6). However these "substitutions" are only used to formulate appropriate inference rules, they do not occur elsewhere in the paper.

\section{Inductive validity and ground confluence}

We assume throughout this paper that $\left(\Sigma^{\wedge}, \mathcal{R}\right)$ is a given constructor based specifcation (induced by $\Sigma=\Sigma_{0}+\Sigma_{1}$ ). To define the basic notions we first introduce the rewrite relation induced by $\left(\Sigma^{\wedge}, \mathcal{R}\right)$.

Definition 3.1 Let $s \longrightarrow_{\mathcal{R}} t$ for $s, t \in \operatorname{TERM}\left(\Sigma^{\wedge}, V\right)$ iff there exists a position $p \in O(s)$, a substitution $\sigma$ (that respects the sort hierarchy restriction) and a rule $u=v \in \mathcal{R}$ such that $s / p \equiv \sigma(u)$ and $t \equiv s[\sigma(v)]_{p}$.

Note that - due to the variable restriction - rewriting has to be performed in a kind of innermost fashion.

One easily proves that $\stackrel{*}{\longleftrightarrow}$ is a congruence relation on $\operatorname{TERM}\left(\Sigma^{\wedge}, V\right)$. Let $\mathcal{T}_{\left(\Sigma^{\wedge}, \mathcal{K}\right)}$ denote the canonical (hierarchical) term algebra induced by the congruence relation $\stackrel{*}{\longrightarrow}$ on $\operatorname{TERM}\left(\Sigma^{\wedge}\right)$. One easily shows that $\mathcal{T}_{\left(\Sigma^{\wedge}, \mathcal{R}\right)}$ is initial in the class of hierarchical $\Sigma^{\wedge}$-algebras that are models of $\mathcal{R}$. So $\mathcal{T}_{\left(\Sigma^{\wedge}, \mathcal{R}\right)}$ represents a kind of constructor semantics of the specification $\left(\Sigma^{\wedge}, \mathcal{R}\right.$ ). In [AvBe92] it is shown (in a more general context including built-in structures) that the above definition of semantics is sound.

Next we define the basic notions this paper is concerned with. In order to make apparent the difference between our constructor approach and the usual one we always emphasize the given order-sorted specification.

Definition $3.2 A \Sigma^{\wedge}$-equation $s=t$ is called an inductive theorem (wrt. $\left(\Sigma^{\wedge}, \mathcal{R}\right)$ ) or inductively valid (wrt. $\left(\Sigma^{\wedge}, \mathcal{R}\right)$ ) iff $\mathcal{T}_{\left(\Sigma^{\wedge}, \mathcal{R}\right)}$ is a model of $s=t$. 
Definition $3.3 \mathcal{R}$ is said to be $\left(\Sigma^{\wedge}\right.$-)ground confluent iff for any terms $s, s_{1}, s_{2} \in$ $\operatorname{TERM}\left(\Sigma^{\wedge}\right)$, if $s_{1} \stackrel{*}{\stackrel{*}{*}} s \stackrel{*}{\longrightarrow} s_{2}$, then $s_{1} \downarrow_{\mathcal{R}} s_{2} \cdot \mathcal{R}$ is said to be locally ( $\Sigma^{\wedge}$-)ground confluent iff $s_{1} \downarrow_{\mathcal{R}} s_{2}$ whenever $s_{1} \longleftarrow s \longrightarrow_{\mathcal{R}} s_{2}$.

Next we relate both notions to ground joinability. This enables us to develop a uniform treatment of inductive validity and ground confluence.

A $\Sigma^{\wedge}$-ground equation $s=t$ is joinable $\left(w r t\right.$. $\left.\left(\Sigma^{\wedge}, \mathcal{R}\right)\right)$ iff $s \downarrow_{\mathcal{R}} t$. An arbitrary $\Sigma^{\wedge}$-equation $s=t$ is ground joinable (wrt. $\left(\Sigma^{\wedge}, \mathcal{R}\right)$ ) iff $\tau(s) \mathfrak{l}_{\mathcal{R}} \tau(t)$ for all ground substitutions $\tau$. Finally, a set $S$ of $\Sigma^{\wedge}$-equations is ground joinable (wrt. $\left(\Sigma^{\wedge}, \mathcal{R}\right)$ ) iff all equations from $S$ are ground joinable (wrt. $\left(\Sigma^{\wedge}, \mathcal{R}\right)$ ).

Lemma 3.1 Let $\mathcal{R}$. be ground confluent. Let $S$ be a set of $\Sigma^{\wedge}$-equations. Then $S$ is inductively valid wrt. $\left(\Sigma^{\wedge}, \mathcal{R}\right)$ iff $S$ is ground joinable wrt. $\left(\Sigma^{\wedge}, \mathcal{R}\right)$.

In order to relate ground confluence to ground joinability we first review the notion of critical pairs. Let $u=v$ and $u^{\prime}=v^{\prime}$ be two rules of $\mathcal{R}$ that share no variables. Let $p$ be a non-variable position of $u$ and let $u / p$ and $u^{\prime}$ be unifiable with most general unifier (mgu) $\mu$. Then $\mu(u)\left[\mu\left(v^{\prime}\right)\right]_{p}=\mu(v)$ is called a critical pair between the two rules. Again note that $\mu$ is a $\Sigma^{\wedge}$-substitution and thus has to respect the sort hierarchy. Let $\operatorname{CRIT}(\mathcal{R})$ denote the set of all critical pairs resulting from the rules of $\mathcal{R}$. The following critical pair lemma is proved just as in the non-constructor case.

Lemma $3.2 \mathcal{R}$ is locally $\Sigma^{\wedge}$-ground confluent iff $C R I T(\mathcal{R})$ is ground joinable wrt. $\left(\Sigma^{\wedge}, \mathcal{R}\right)$.

By Newmans lemma, ground confuence and local ground confluence are equivalent, provided $\mathcal{R}$ is (ground) terminating, i.e. $\stackrel{+}{\mathcal{R}}_{\text {is a }}$ is well-founded relation on $\operatorname{TERM}\left(\Sigma^{\wedge}\right)$.

To guarantee the latter, we assume that we are given a reduction ordering $>$ on $\operatorname{TERM}(\Sigma, V)$ (and thus on $\operatorname{TERM}\left(\Sigma^{\wedge}, V\right)$ ) such that $\mathcal{R}$ is compatible with $>$, i.e. $u>v$ for any $u=v \in \mathcal{R}$. Some considerations to be made below require orderings that in addition have the subterm property. For that reason we assume that $>$ is ground subtcrm compatible, meaning that there exists a well-founded partial ordering $\succ$ on $\operatorname{TERM}(\Sigma)$ such that (i) for all $s, t \in \operatorname{TERM}(\Sigma)$, if $s>t$ then $s \succ t$ and (ii) $s[t] \succ t$ for all $s, t \in T E R M(\Sigma)$ with $s \neq t$. Then, as a consequence, the following statement needed below is true: if $s_{1} \longleftarrow s \longrightarrow_{R} s_{2}$, then $s_{1} \downarrow_{\pi} s_{2}$, or there exists a critical pair $u=v$ and a ground substitution $\tau$ such that $\{s\} \succ \succ \tau(u), \tau(v)\}$.

\section{Ground reducibility}

The prover to be developed below is based on the notion of ground reducibility.

Definition $4.1 A \Sigma^{\wedge}$-term $t$ is said to be ground reducible (wrt. $\left(\Sigma^{\wedge}, \mathcal{R}\right)$ ) iff $\tau(t)$ is reducible by $\mathcal{R}$ for any ground substitulion $\tau$.

Ground reducibility is decidable in the flat case (see [Pl85, KNZ87]). We show that this result carries over to the constructor based approach (without taking efficiency into consideration). 
In the constructor based setting instantiation of a variable by a term involving a function symbol of $F_{1}$ is not allowed. We introduce a new flat setting where the symbols of $F_{1}$ are replaced in a certain sense by new function symbols that map into a new sort. The variable restriction then can be modeled using the requirement of flat sort compatibility.

First let $t$ be an arbitrary $\Sigma^{\wedge}$-term. A maximal $\Sigma_{0}$-subterm of $t$ is a subterm $t / p \in \operatorname{TERM}_{0}(\Sigma, V)$ of $t$ such that $t / p^{\prime}$ contains a new symbol of $F_{1}$ for any position $p^{\prime}$ above $p$. Let $w_{1}, \ldots, w_{n}$ be the sequence of the maximal $\Sigma_{0}$-subterms of $t$ in the "natural ordering from left to right" (i.e. if $i<j$ and $w_{i} \equiv t / p_{i}$ as well as $w_{j} \equiv t / p_{j}$, then $p_{i}<_{\text {lex }} p_{j}$, where $<_{\text {lex }}$ is the usual lexicographic ordering). Note that two terms that are unifiable have the same number of maximal $\Sigma_{0}$-subterms.

Now let $t$ be a given $\Sigma^{\wedge}$-term. We construct a new signature $\Sigma^{\#}$, which is a flat enrichment of $\Sigma_{0}$, a new $\Sigma^{\#}$-term $t^{\$}$ and a new set $\mathcal{R}^{\#}$ of rules over $\Sigma^{\#}$ such that $t$ is ground reducible wrt. $\left(\Sigma^{\wedge}, \mathcal{R}\right)$ iff $t^{\$}$ is ground reducible wrt. $\left(\Sigma^{\#}, \mathcal{R}_{0} \cup \mathcal{R}^{\#}\right)$.

Let $t_{1}, \ldots, t_{k}$ be the $\Sigma^{\wedge}$-subterms of $t$ that are unifiable with a left hand side of a rule of $\mathcal{R}_{1}$. In particular let $t_{i}$ be unifiable with $u_{i}$ where $u_{i}=v_{i} \in \mathcal{R}_{1}$. Further let $t_{0} \equiv t$.

In a first step we define the new signature. Let $s_{\#}$ be a new sort symbol and

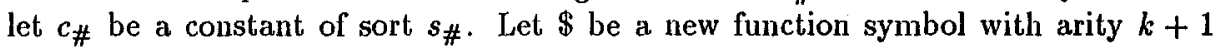
with declaration $\$: s_{\#}, \ldots, s_{\#} \longrightarrow s_{\#}$. For any $i \in\{0, \ldots, k\}$ let $w_{i 1}, \ldots, w_{i n_{i}}$ be the sequence of maximal $\Sigma_{0}$-subterms of $t_{i}$. Let $\#_{i}(i=0, \ldots, k)$ be a new function symbol

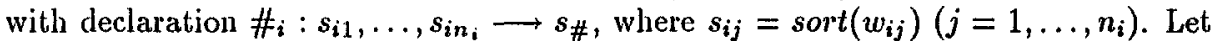
$\Sigma^{\#}$ result from $\Sigma_{0}$ by adding all the new symbols.

Next we construct the $\Sigma^{\#-t e r m} t^{\$}$ and the set $\mathcal{R}^{\#}$ of $\Sigma^{\#-r u l e s . ~ L e t ~} t_{i}^{\#} \equiv$ $\#_{i}\left(w_{i 1}, \ldots, w_{i n_{i}}\right)(i=0, \ldots, k)$. Analogously, let $u_{i}^{\#} \equiv \#_{i}\left(w_{i 1}^{\prime}, \ldots, w_{i n_{i}}^{\prime}\right)(i=$ $1, \ldots, k)$, where $w_{i 1}^{\prime}, \ldots, w_{i n_{i}}^{\prime}$ is the sequence of maximal $\Sigma_{0}$-subterms of $u_{i}$. Now let $t^{\$} \equiv \$\left(\iota_{0}^{\#}, \ldots, t_{k}^{\#}\right)$ and let $\mathcal{R}^{\#}=\left\{u_{i}^{\#}=c_{\#} \mid i=1, \ldots, k\right\}$.

Using the following lemma as well as the decidability result concerning the usual unstructured setting we obtain decidability of ground reducibility in the constructor based setting.

Lemma 4.1 The hierarchical $\Sigma^{\wedge}$-term $t$ is ground reducible wrt. $\left(\Sigma^{\wedge}, \mathcal{R}\right)$ iff the flat

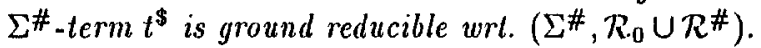

To illustrate the above considerations we continue our example from the introduction. Let $\mathcal{R}_{0}$ be empty and $\mathcal{R}_{1}$ consist of the rules $(R 1) \ldots(R 4)$. Let $t \equiv$ $(x-y)+y$. Then $t^{\$} \equiv \$\left(\#_{0}(x, y, y), \#_{1}(x, y), \#_{2}(x, y)\right)$ and $\mathcal{R}^{\#}=\left\{_{1}(x, 0)=\right.$ $c_{\#} ; \#_{2}(s(x), s(y))=c_{\#}$. Now $t^{\$}$ is not ground reducible wrt. $\mathcal{R}^{\#}$. Take for instance the ground substitution that instantiates $x$ by 0 and $y$ by $s(0)$. Using the lemma one obtains that $t$ is not ground reducible wrt. $\mathcal{R}$ in the hierachical setting.

Next we consider ground reducibility of $\Sigma^{\wedge}$-equations.

Definition $4.2 A \Sigma^{\wedge}$-equation $s=t$ is ground reducible wrt. $\left(\Sigma^{\wedge}, \mathcal{R}\right)$ iff for any ground substitution $\tau$, if $\tau(s) \neq \tau(t)$, then $\tau(s)$ or $\tau(t)$ is reducible by $\mathcal{R}$.

Ground reducibility of an equation $s=t$ is usually encoded by introducing a new symbol $\approx$ and a new rule with left hand side $\approx(x, x)$ and considering the "new term" $s \approx t$. In our context we have to respect the variable restriction. Let $\approx$, be a new 
function symbol and $T_{s}$ be a new constant symbol for any sort $s \in S_{0}^{\wedge}$. We drop the sort subscripts for simplicity. Let $\Sigma_{\approx}^{\wedge}$ result from $\Sigma^{\wedge}$ by adding the new symbols. Finally let $\mathcal{R}_{s=t}$ be the set $\mathcal{R} \cup\{\mu(s) \approx \mu(t)=T\}$ in the case where $s$ and $t$ are unifiable with mgu $\mu$ and let $\mathcal{R}_{s=t}$ be the set $\mathcal{R}$ otherwise. The following lemma shows that ground reducibility of equations as well is decidable.

Lemma 4.2 The $\Sigma^{\wedge}$-equation $s=t$ is ground reducible wrt. $\left(\Sigma^{\wedge}, \mathcal{R}\right)$ iff the $\Sigma_{\approx}^{\wedge}$-term $s \approx t$ is ground reducible wrt. $\left(\Sigma^{\wedge}, \mathcal{R}_{s=t}\right)$.

\section{The prover}

In order to make transparent the argumentations to follow, we separate the basic concepts of the proof procedure from the technical details by introducing some new terminology. This separation is also convenient for another reason. We plan to extend the ideas of the present paper to conditional equational specifications by keeping the frame of the prover. So we only have to change the technical details. They become much more complicated in the conditional case as we have to circumvent ground reducibility, which is known to be undecidable in that case (see [KaCh86]).

The present section can be divided into two parts. In the first one we describe the kind of objects the prover operates on. Then, in a second part, we give a description of the prover from an abstract point of view.

Our aim is to prove ground joinability of a given set $\mathcal{C}$ of equations. The elements of $\mathcal{C}$ are to be transformed by some inference rules until ground joinability or unjoinability (possibly) becomes decidable.

In order to enable strong inductive reasoning we enrich the equations to be transformed by so-called $\mathcal{C}$-references (see also [Wi91]). A $\mathcal{C}$-reference $(C, \rho)$ consists of an element $C$ of $\mathcal{C}$ and a substitution $\rho$. An equation with $\mathcal{C}$-reference (or simply an equation) is a pair $(s=t,(C, \rho))$ consisting of an "ordinary" $\Sigma^{\wedge}$-equation $s=t$ and a $\mathcal{C}$-reference $(C, \rho)$. Semantically, the equation $s=t$ should be considered as the result of a transformation of the instance $\rho(C)$. We identify an equation $s=t$ with the corresponding pair $(s=t,(s=t, i d))$.

An equation $(s=t,(C, \rho))$ is ground joinable $\left(w r t .\left(\Sigma^{\wedge}, \mathcal{R}\right)\right)$ iff $s=t$ is ground joinable (wrt. $\left.\left(\Sigma^{\wedge}, \mathcal{R}\right)\right)$. An unjoinability witness $($ for a set $S)\left(\right.$ wrt. $\left(\Sigma^{\wedge}, \mathcal{R}\right)$ ) is a ground instance $((s=t,(C, \rho)), \tau)$ of an equation $(s=t,(C, \rho))$ (of $S)$ such that $\tau(s)=\tau(t)$ is not joinable (wrt. $\mathcal{R})$.

In order to be able to formulate some of the basic properties of the prover we next introduce appropriate complexity measures. Let $\succ$ be a well-founded ordering on the ground terms that has the proper subterm property and extends $>$ (see section 3). To measure (ordinary) equation instances let $m(s=t, \tau)=\{\tau(s), \tau(t)\}$ and $\succ_{e}=\succ \succ$. Further, (referenced) equation instances are measured according to $m((s=t,(C, \rho)), \tau)=(m(C, \tau \rho), m(s=t, \tau))$ and $\succ_{r}$, where $\succ_{r}$ is the lexicographical combination of $\succ_{e}$ and $\succ_{e}$.

Next we introduce a notion that is needed to prove ground confluence. An equation (with reference) $(s=t,(C, \rho))$ is said to be admissible iff $m(C, \tau \rho) \succeq_{e} m(s=t, \tau)$ for any ground substitution $\tau$. A set of equalions is admissible iff all its elements are admissible. To explain the reason for introducing this notion let $(s=t,(C, \rho))$ be an equation and $\tau$ a ground substitution such that (e.g.) $\tau(s)$ gives rise to a 
divergency $s_{1}{ }_{\mathcal{R}}^{\leftarrow} \tau(s) \longrightarrow_{\mathcal{R}} s_{2}$. Then there exists a related critical pair instance $\left(u=v, \tau^{\prime}\right)$ which we want to be "smaller" than the original reference $(C, \tau \rho)$. As $m(s=t, \tau) \succ_{e} m\left(u=v, \tau^{\prime}\right)$ (see section 3 ) we get the desired property provided the equation $(s=t,(C, \rho))$ is admissible.

The prover we are going to describe now is based on the concepts developed in [Ba88]. We present an inference system $\mathcal{I}$ that operates on admissible equations (with $\mathcal{C}$-references). For technical reasons we split these equations into two groups, $\mathcal{H}$ and $\mathcal{G} . \mathcal{H}$ contains the actual hypotheses and $\mathcal{G}$ contains some equations that are stored in order to be available for inductive steps. 'Thus, the inference rules operate on pairs $(\mathcal{G}, \mathcal{H})$ of sets of equations with $\mathcal{C}$-references. As usual we write $(\mathcal{G}, \mathcal{H}) \vdash_{\mathcal{I}}\left(\mathcal{G}^{\prime}, \mathcal{H}^{\prime}\right)$ if $\left(\mathcal{G}^{\prime}, \mathcal{H}^{\prime}\right)$ results from $(\mathcal{G}, \mathcal{H})$ by applying an inference rule on $(\mathcal{G}, \mathcal{H})$. The inference rules themselves will be formulated in the next section. The rest of this section deals with some invariants of the inference system that imply the main properties of the prover.

We say that $\mathcal{H}$ is a covering set for $\mathcal{G}$ iff the following condition holds: if $(G, \tau)$ is an unjoinability witness for $\mathcal{G}$, then $(G, \tau)$ is an unjoinability witness for $\mathcal{H}$, or there exists an unjoinability witness $\left(H, \tau^{\prime}\right)$ for $\mathcal{H}$ with $m(G, \tau) \succ_{r} m\left(H, \tau^{\prime}\right)$. A pair $(\mathcal{G}, \mathcal{H})$ preserves admissibility resp. joinability iff $\mathcal{H}$ is admissible resp. ground joinable whenever $\mathcal{G}$ is. It preserves unjoinability if $\mathcal{H}$ is a covering set for $\mathcal{G}$.

An inference step $(\mathcal{G}, \mathcal{H}) \vdash_{\mathcal{I}}\left(\mathcal{G}^{\prime}, \mathcal{H}^{\prime}\right)$ preserves admissibility resp. unjoinability iff the pair $\left(\mathcal{H}, \mathcal{H}^{\prime}\right)$ preserves the according property. An inference step $(\mathcal{G}, \mathcal{H}) \vdash_{\mathcal{I}}\left(\mathcal{G}^{\prime}, \mathcal{H}^{\prime}\right)$ preserves joinability iff the pair $\left(\mathcal{G} \cup \mathcal{H}, \mathcal{G}^{\prime} \cup \mathcal{H}^{\prime}\right)$ preserves joinability. A derivation then preserves a property iff all the inference steps preserve the property. An inference step $(\mathcal{G}, \mathcal{H}) \vdash_{\mathcal{I}}\left(\mathcal{G}^{\prime}, \mathcal{H}^{\prime}\right)$ inductively preserves unjoinability iff the following condition holds: if $(H, \tau)$ is an unjoinability witness for $\mathcal{H}$, then $(H, \tau)$ is an unjoinability witness for $\mathcal{H}^{\prime}$, or there exists an unjoinability witness $\left(H^{\prime}, \tau^{\prime}\right)$ for $\mathcal{G}^{\prime} \cup \mathcal{H}^{\prime}$ with $m(H, \tau) \succ_{r} m\left(H^{\prime}, \tau^{\prime}\right)$. Finally, an inference step $(\mathcal{G}, \mathcal{H}) \vdash_{I}\left(\mathcal{G}^{\prime}, \mathcal{H}^{\prime}\right)$ is generic iff $\mathcal{G}^{\prime} \subseteq \mathcal{G} \cup \mathcal{H}$.

Lemma 5.1 Let $(\mathcal{G}, \mathcal{H})$ prescrve unjoinability. Let $(\mathcal{G}, \mathcal{H}) \vdash_{\mathcal{I}}\left(\mathcal{G}^{\prime}, \mathcal{H}^{\prime}\right)$ be an inference step that inductively preserves unjoinability and that is generic. Then the inference step preserves unjoinability.

First we consider "positive proofs". Note that we only have to require the derivation to preserve unjoinability.

Lemma 5.2 Let the pair $(\mathcal{G}, \mathcal{H})$ as well as the derivation $(\mathcal{G}, \mathcal{H}) \vdash_{\mathcal{I}} \cdots \vdash_{\mathcal{I}}\left(\mathcal{G}^{\prime}, \mathcal{H}^{\prime}\right)$ preserve unjoinability. Let $\mathcal{H}^{\prime}=\emptyset$. Then $\mathcal{G}$ is ground joinable.

In order to make possible "negative proofs" or refutations we have to introduce a failure predicate $f a i l(\mathcal{H})$. It will be made precise in the next section. Here we only use the correctness of the predicate. The Cailure predicate fail is correct wrt. joinability iff $f a i l(\mathcal{H})$ is false whenever $\mathcal{H}$ is ground joinable.

Lemma 5.3 Let the pair $(\mathcal{G}, \mathcal{H})$ as well as the derivation $(\mathcal{G}, \mathcal{H}) \vdash_{\mathcal{I}} \cdots \vdash_{\mathcal{I}}\left(\mathcal{G}^{\prime}, \mathcal{H}^{\prime}\right)$ preserve joinability. Let fail be correct wrt. joinability. Let fail $\left(\mathcal{H}^{\prime}\right)$ be true. Then $\mathcal{G}$ is not ground joinable.

Refutational completeness of the prover requires the derivations to be fair. A sequence $\left(\mathcal{G}_{0}, \mathcal{H}_{0}\right),\left(\mathcal{G}_{1}, \mathcal{H}_{1}\right), \ldots$ represents a fair derivation wrt. fail iff either there 
exists an index $k$ such that $\left(\mathcal{G}_{0}, \mathcal{H}_{0}\right) \vdash_{\mathcal{I}} \cdots \vdash_{\mathcal{I}}\left(\mathcal{G}_{k}, \mathcal{H}_{k}\right)$ and $f a i l\left(\mathcal{H}_{k}\right)$ is true, or for all $k$ we have $\left(\mathcal{G}_{k}, \mathcal{H}_{k}\right) \vdash_{I}\left(\mathcal{G}_{k+1}, \mathcal{H}_{k+1}\right)$ and $\bigcup_{i} \bigcap_{j \geq i} \mathcal{H}_{j}=\emptyset$.

Lemma 5.4 Let the pair $(\mathcal{G}, \mathcal{H})$ preserve unjoinability. Let the sequence $(\mathcal{G}, \mathcal{H})=$ $\left(\mathcal{G}_{0}, \mathcal{H}_{0}\right),\left(\mathcal{G}_{1}, \mathcal{H}_{1}\right), \ldots$ represent a fair derivation that as well preserves unjoinability. If $\mathcal{G}$ is not ground joinable, then there exists an index $k$ such that fail( $\left.\mathcal{H}_{k}\right)$ is true.

In order to assure the existence of fair derivations we introduce the following notion. The inference system $\mathcal{I}$ allows continuation wrt. fail iff for any $H \in \mathcal{H}$, if fail $(H)$ is not true, then there exists a set $\mathcal{H}^{\prime}$ such that $(\mathcal{G}, \mathcal{H}) \vdash_{\mathcal{I}}\left(\mathcal{G}^{\prime}, \mathcal{H}^{\prime}\right)$ and $H \notin \mathcal{H}^{\prime}$.

Lemma 5.5 Let $\mathcal{I}$ allow continuation wrt. fail. Then for any $(\mathcal{G}, \mathcal{H})$ there exists a sequence $(\mathcal{G}, \mathcal{H})=\left(\mathcal{G}_{0}, \mathcal{H}_{0}\right),\left(\mathcal{G}_{1}, \mathcal{H}_{1}\right), \ldots$ that represents a fair derivation wrt. fail.

\section{The inference system}

In section 5 we have developed the basic concepts of our prover. Here we show the details: We make precise the inference system $\mathcal{I}$, we define the failure predicate fail, and we prove the properties to make applicable the lemmas of section 5 . That will immediately define our proof procedure.

The first rule uses superposition in order to propagate hypotheses. Let $H:(s=$ $\ell,(C, \rho)$ ) be an equation (with $\mathcal{C}$-reference) and $u=v$ a rule of $\mathcal{R}$ such that $H$ and $u=v$ share no variables. If $s / p$ ( $t / p$ is treated analogously) and $u$ are unifiable with $\operatorname{mgu} \mu$ and if $s / p$ is not a variable, then $\left(\mu(s)[\mu(v)]_{p}=\mu(t),(C, \mu \rho)\right)$ is a superposition result between $H$ and $\mathcal{R}$ (at the position $p)$. Let $S U P(H, \mathcal{R})(\operatorname{resp} S U P(H, p, \mathcal{R}))$ be the set of all superposition results between $H$ and $\mathcal{R}$ (al the position $p$ ).

Rule 1 (Superposition) Let $\mathcal{S} \subseteq S U P(H, \mathcal{R})$ be a covering set for $\{H\}$. Then

$$
\frac{(\mathcal{G}, \mathcal{H} \cup\{H\})}{(\mathcal{G} \cup\{H\}, \mathcal{H} \cup \mathcal{S})}
$$

As every equation of $\mathcal{H}$ has to be treated by the inference system, the set $\mathcal{S} \subseteq$ $S U P(H, \mathcal{R})$ of superposition results should be kept small. In many cases it suffices to superpose at a "complete" single position: Let $H:(s=t,(C, \rho))$. A position $p \in O(s)$ (or $p \in O(t)$ ) is said to be complete wrt. $\mathcal{R}$ iff $S U P(H, p, \mathcal{R})$ is a covering set for $H$.

Next we make precise the fail-predicate exactly in the same way as in [Ba88].

Definition 6.1 For $H:(s=t,(C, \rho))$ let fail $(H)$ be true iff either $s>t$ and $s$ is not ground reducible wrt. $\left(\Sigma^{\wedge}, \mathcal{R}\right)$, or $t>s$ and $t$ is not ground reducible wrt. $\left(\Sigma^{\wedge}, \mathcal{R}\right)$, or $s<>t$ and $s=t$ is not ground reducible wrt. $\left(\Sigma^{\wedge}, \mathcal{R}\right)$. Let fail $(\mathcal{H})$ be true iff there exists a hypothesis $H \in \mathcal{H}$ such that fail( $H)$ is true.

One easily proves that fail is correct wrt. joinability. The following lemma shows that $\mathcal{I}$ allows continuation wrt. fail, provided Rule 1 is a member of $\mathcal{I}$.

Lemma 6.1 If fail( $(H)$ is falsc, then $S U P(H, \mathcal{R})$ is a covering set for $\{H\}$. 
The simplification and deletion rules to be defined below use instantiation of equations. These equations may be rules from $\mathcal{R}$, hypotheses from $\mathcal{G}$ or lemmata from $\mathcal{L}$, where $\mathcal{L}$ is a set of equations which are known to be ground joinable. Instantiation by substitutions is too weak a concept, as substitutions have to respect the sort hierarchy and so variables can only be replaced by terms without new function symbols. In order to get rid of this restriction we introduce what we call quasi-substitutions.

Definition $6.2 A \Sigma^{\wedge}$-term $t$ is said lo be $\Sigma_{0}$-defined (wrt. $\left(\Sigma^{\wedge}, \mathcal{R}\right)$ ) iff for any ground substilution $\tau$ there exists an element $w$ in $T E R M_{0}\left(\Sigma^{\wedge}\right)$ such that $\tau(t){\stackrel{*}{{ }_{R}}} w$.

In section 7 we show how to test whether a term is $\Sigma_{0}$-defined.

Definition 6.3 A mapping $\sigma: V \rightarrow \operatorname{TERM}\left(\Sigma^{\wedge}, V\right)$ is said to be a quasi-substitution wrt. $\left(\Sigma^{\wedge}, \mathcal{R}\right)$ iff (i) $\operatorname{DOM}(\sigma)=\{x \mid \sigma(x) \neq x\}$ is finite, (ii) $\operatorname{sort}(x) \unlhd \operatorname{sort}(\sigma(x))$ for all $x \in D O M(\sigma)$ and (iii) $\sigma(x)$ is $\Sigma_{0}$-defincd wrt. $\left(\Sigma^{\wedge}, \mathcal{R}\right)$ for any $x \in D O M(\sigma)$.

Rule 2 (Simplification) Let $H:(s=t,(C, \rho))$. Let $u=v$ be an equation with $u=v \in \mathcal{R} \cup \mathcal{L}$ or $\left(u=v,\left(C^{\prime}, \rho^{\prime}\right)\right) \in \mathcal{G}$. Let $p \in O(s)$ (analogously for $t$ ) and $\sigma$ be a quasi-substilution wrt. $\left(\Sigma^{\wedge}, \mathcal{R}\right.$ ) such that (i) $s / p \equiv \sigma(u)$, (ii) $\sigma(u)>\sigma(v)$ and (iii) $\rho(C)>>\sigma \rho^{\prime}\left(C^{\prime}\right)$ provided $\left(u=v,\left(C^{\prime}, \rho^{\prime}\right)\right) \in \mathcal{G}$. Let $H^{\prime}:\left(s[\sigma(v)]_{p}=t,(C, \rho)\right)$. Then

$$
\frac{(\mathcal{G}, \mathcal{H} \cup\{H\})}{\left(\mathcal{G}, \mathcal{H} \cup\left\{H^{\prime}\right\}\right)}
$$

To be able to formulate a quite general deletion rule we first introduce two new notations. First, two $\Sigma^{\wedge}$-terms $s$ and $t$ are quasi-equationally related by $\mathcal{R} \cup \mathcal{G} \cup \mathcal{L}$ below $(C, \rho)$ iff there exists an equation $u=v$ with $u=v \in \mathcal{R} \cup \mathcal{L}$ or $\left(u=v,\left(C^{\prime}, \rho^{\prime}\right)\right) \in \mathcal{G}$ and a quasi-substitution $\sigma$ wrt. $\left(\Sigma^{\wedge}, \mathcal{R}\right)$ such that (i) $s \equiv w[\sigma(u)]_{p}$ and $t \equiv w[\sigma(v)]_{p}$ (for appropriate $w$ and $p$ ) and (ii) $\rho(C)>>\sigma \rho^{\prime}\left(C^{\prime}\right)$ provided $\left(u=v,\left(C^{\prime}, \rho^{\prime}\right)\right) \in \mathcal{G}$. Second, two $\Sigma^{\wedge}$-terms $s$ and $t$ are quasi-subconnected by $\mathcal{R} \cup \mathcal{G} \cup \mathcal{L}$ below $(C, \rho)$ iff there exist a number $n \geq 0$ and terms $s_{0}, \ldots, s_{n} \in \operatorname{TERM}\left(\Sigma^{\wedge}, V\right)$ such that (i) $s \equiv s_{0}$ and $t \equiv s_{n}$, (ii) $s_{i}$ and $s_{i+1}$ are quasi-equationally related by $\mathcal{R} \cup \mathcal{G} \cup \mathcal{L}$ below $(C, \rho)$ for $i=0, \ldots, n-1$ and (iii) $\rho(C) \gg\left\{s_{i}\right\}$ for $i=1, \ldots, n-1$.

Rule 3 (Deletion) Let $H:(s=t,(C, \rho))$. Let $s$ and $t$ be quasi-subconnected by $\mathcal{R} \cup \mathcal{G} \cup \mathcal{L}$ below $(C, \rho)$. Then

$$
\frac{(\mathcal{G}, \mathcal{H} \cup\{H\})}{(\mathcal{G}, \mathcal{H})}
$$

The following deletion rules (deletion of trivial equations and deletion by subsumption) can be obtained as special cases of Rule 3 .

Rule 4 (Equality Check) Let $H:(s=t,(C, \rho))$ be such that $s \equiv t$. Then

$$
\frac{(\mathcal{G}, \mathcal{H} \cup\{H\})}{(\mathcal{G}, \mathcal{H})}
$$

Rule 5 (Subsumption) Let $H:(s=t,(C, \rho))$. Let $s$ and $t$ be quasi-equationally related by $\mathcal{R} \cup \mathcal{G} \cup \mathcal{L}$ bclow $(C, \rho)$. Then 


$$
\frac{(\mathcal{G}, \mathcal{H} \cup\{H\})}{(\mathcal{G}, \mathcal{H})}
$$

The following lemma sums up all the "correctness properties" of the rules. Let $I$ consist of Rule 1 , Rule 2 and Rule 3.

\section{Lemma 6.2}

(a) Every inference step $(\mathcal{G}, \mathcal{H}) \vdash_{\mathcal{I}}\left(\mathcal{G}^{\prime}, \mathcal{H}^{\prime}\right)$ is generic.

(b) Every inference slep $(\mathcal{G}, \mathcal{H}) \vdash_{\mathcal{I}}\left(\mathcal{G}^{\prime}, \mathcal{H}^{\prime}\right)$ preserves admissibility.

(c) Let $\mathcal{R}$ be ground confluent and let $\mathcal{G}$ be ground joinable. Then every inference step $(\mathcal{G}, \mathcal{H}) \vdash_{\mathcal{I}}\left(\mathcal{G}^{\prime}, \mathcal{H}^{\prime}\right)$ preserves joinability.

(d) Let $\mathcal{R}$ be ground conflucnt or $\operatorname{CRIT}(\mathcal{R}) \subseteq \mathcal{G}$. Let $\mathcal{H}$ be admissible. Then every inference sicp $(\mathcal{G}, \mathcal{H}) \vdash_{\mathcal{I}}\left(\mathcal{G}^{\prime}, \mathcal{H}^{\prime}\right)$ inductively preserves unjoinability.

\section{$7 \quad$ Results and examples}

In this section we present our proof procedures and illustrate them by examples. In theorem 7.1 we show how to prove $\Sigma^{\wedge}$-ground confluence of $\mathcal{R}$.

\section{Theorem 7.1}

(a) Let $\operatorname{CRIT}(\mathcal{R}) \subseteq \mathcal{C}$. If $(\mathcal{C}, \mathcal{C}) \vdash_{\mathcal{I}} \cdots \vdash_{\mathcal{I}}\left(\mathcal{G}^{\prime}, \emptyset\right)$, then $\mathcal{R}$ is $\Sigma^{\wedge}$-ground confluent.

(b) Let $\mathcal{C}=\operatorname{CRIT}(\mathcal{R})$. If $(\mathcal{C}, \mathcal{C}) \vdash_{\mathcal{I}} \cdots \vdash_{\mathcal{I}}\left(\mathcal{G}^{\prime}, \mathcal{H}^{\prime}\right)$ such that fail $\left(\mathcal{H}^{\prime}\right)$ is true, then $\mathcal{R}$ is not $\Sigma^{\wedge}$-ground confluent.

(c) Let $\mathcal{C}=\operatorname{CRIT}(\mathcal{R})$. If $(\mathcal{C}, \mathcal{C})=\left(\mathcal{G}_{0}, \mathcal{H}_{0}\right),\left(\mathcal{G}_{1}, \mathcal{H}_{1}\right), \ldots$ represents a fair derivation wrt. fail and if $\mathcal{R}$ is not $\Sigma^{\wedge}$-ground confluent, then there exists an index $k$ such that fail $\left(\mathcal{H}_{k}\right)$ is true.

The following example is taken from [NRS89]. Note again that all terms have to be interpreted hierarchically and that variables are of a "low sort".

Example 7.1 Let $F_{0}=\{0, s, p\}$ be the set of constructors and $F_{1}=\{+,-, *\}$ the set of new symbols. Further let $\mathcal{R}_{0}=\{R 1, R 2\}$ define integers and let $\mathcal{R}_{1}=$ $\{R 3, \ldots, R 11\}$.

$$
\begin{aligned}
& (R 1) s(p(x))=x \\
& (R 2) p(s(x))=x \\
& (R 3) \quad x+0=x \\
& (R 4) x+s(y)=s(x+y) \\
& (R 5) \quad x+p(y)=p(x+y)
\end{aligned}
$$

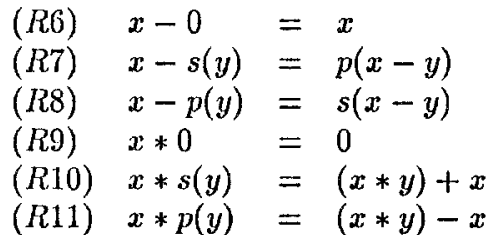

We obtain the following set $C R I T(\mathcal{R})=\{C 1, \ldots, C 8\}$ of critical pairs: 


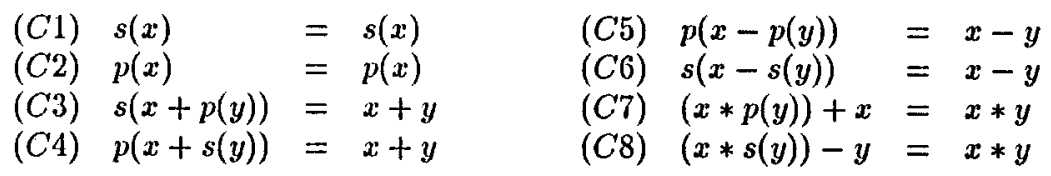

In order to show that $\mathcal{R}$ is ground confluent, we add the following equations $\mathcal{A}=$ $\{A 1, \ldots, A 6\}$ :

$$
\begin{aligned}
& \text { (A1) } p(x)+y \cdot=p(x+y) \\
& \text { (A2) } s(x)+y=s(x+y) \\
& \text { (A4) } p(x)-y=p(x-y) \\
& \text { (A3) }(x-y)+y=x \\
& \text { (A5) } s(x)-y=s(x-y) \\
& \text { (A6) }(x+y)-y=x
\end{aligned}
$$

Now, using the fact that all $\Sigma^{\wedge}$-terms are $\Sigma_{0}$-defined wrt. $\left(\Sigma^{\wedge}, \mathcal{R}\right)$, we easily show that $(\mathcal{C}, \mathcal{C}) \vdash_{\mathcal{I}}\left(\mathcal{G}^{\prime}, \emptyset\right)$ where $\mathcal{C}=\operatorname{CRIT}(\mathcal{R}) \cup \mathcal{A}$. Thus $\mathcal{R}=\mathcal{R}_{0}+\mathcal{R}_{1}$ is $\Sigma^{\wedge}$-ground confluent (in the constructor approach). In addition we obtain by the following theorem that the equations in $\mathcal{A}$ are inductively valid wrt. $\left(\Sigma^{\wedge}, \mathcal{R}\right)$.

Next we turn to inductive theorem proving.

\section{Theorem 7.2}

(a) Let $\mathcal{R}$ be ground confluent. If $(\mathcal{C}, \mathcal{C}) \vdash_{T} \cdots \vdash_{\mathcal{I}}\left(\mathcal{G}^{\prime}, \emptyset\right)$, then $\mathcal{C}$ is inductively valid wrt. $\left(\Sigma^{\wedge}, \mathcal{R}\right)$.

(b) Let $\mathcal{R}$ be ground confluent. If $(\mathcal{C}, \mathcal{C}) \vdash_{\mathcal{I}} \cdots \vdash_{\mathcal{I}}\left(\mathcal{G}^{\prime}, \mathcal{H}^{\prime}\right)$ such that fail( $\left.\mathcal{H}^{\prime}\right)$ is true, then $\mathcal{C}$ is nol inductively valid wrt. $\left(\Sigma^{\wedge}, \mathcal{R}\right)$.

(c) Let $\mathcal{R}$ be ground confluent. If $(\mathcal{C}, \mathcal{C})=\left(\mathcal{G}_{0}, \mathcal{H}_{0}\right),\left(\mathcal{G}_{1}, \mathcal{H}_{1}\right), \ldots$ represents a fair derivation wri. fail and if $\mathcal{C}$ is not inductively valid wrt. $\left(\Sigma^{\wedge}, \mathcal{R}\right)$, then there exists an index $k$ such that fail $\left(\mathcal{H}_{k}\right)$ is true.

Using preservingness properties that are based on inductive validity instead of joinability we can even drop the condition of $\mathcal{R}$ being ground confluent in the "positive case (a)" of theorem 7.2 .

Theorem 7.3 If $(\mathcal{C}, \mathcal{C}) \vdash_{\mathcal{I}} \cdots \vdash_{\mathcal{I}}\left(\mathcal{G}^{\prime}, \emptyset\right)$, then $\mathcal{C}$ is inductively valid wrt. $\left(\Sigma^{\wedge}, \mathcal{R}\right)$

We again consider the example introduced in the introduction.

Example 7.2 Let $F_{0}=\{0, s\}$ and $F_{1}=\{+,-\}$. Let $\mathcal{R}_{0}=\emptyset$ and $\mathcal{R}_{1}=\{R 1, \ldots, R 4\}$. $\mathcal{R}$ is $\Sigma^{\wedge}$-ground confluent as $\operatorname{CRIT}(\mathcal{R})=\emptyset$.

$$
\begin{array}{llll}
(R 1) x+0 & =x & (R 3) x-0 & =x \\
(R 2) \quad x+s(y) & =s(x+y) & (R 4) s(x)-s(y) & =x-y
\end{array}
$$

We analyse whether the following hypotheses are inductively valid wrt. $\left(\Sigma^{\wedge}, \mathcal{R}\right)$ or not. The references will be dropped if we do not have to take them into consideration.

$$
\begin{array}{lll}
(H 1) & (x+y)-y=x & (H 2)(x-y)+y=x
\end{array}
$$

To show that $H 1:(x+y)-y=x$ is an inductive theorem wrt. $\left(\Sigma^{\wedge}, \mathcal{R}\right)$ we first use superposition. Note that by the variable restriction we only have to overlap with rule $(R 1)$ and $(R 2)$. The superposition results are (i) $x-0=x$, which can be further 
simplified and then deleled, and (ii) $\left(s\left(x^{\prime}+y^{\prime}\right)-s\left(y^{\prime}\right)=x^{\prime},((x+y)-y=x,\{x \leftarrow\right.$ $\left.\left.\left.x^{\prime}, y \leftarrow s\left(y^{\prime}\right)\right\}\right)\right)$. The latter hypothesis can be simplified by (R4) using the fact that $x^{\prime}+y^{\prime}$ is $\Sigma_{0}$-defined. The resull $\left(\left(x^{\prime}+y^{\prime}\right)-y^{\prime}=x^{\prime},\left((x+y)-y=x,\left\{x \leftarrow x^{\prime}, y \leftarrow\right.\right.\right.$ $\left.\left.\left.s\left(y^{\prime}\right)\right\}\right)\right)$ then can be deleted by the subsumption rule.

The hypothesis $H 2:(x-y)+y=x$ can directly be refuted, as $(x-y)+y>x$ (by an appropriate ordering $>$ ) and $(x-y)+y$ is not ground reducible (see section 4).

The following result (obtained by J. Avenhaus) shows that our prover as well can be used to show that a term is $\Sigma_{0}$-defined. This fact is needed to apply Rule 2 and Rule 3. For that purpose we introduce new constants $*_{s}$ for all $s \in S_{0}$. Let $\Sigma_{*}^{\wedge}$ be the resulting signature. For simplicity we drop the sort subscripts. Let $\mathcal{R}^{*}=$ $\left\{f\left(x_{1}, \ldots, x_{n}\right)=* \mid f \in F_{0}\right\}$.

Lemma 7.1 Let TERM $\left(\Sigma^{\wedge}\right) \neq \emptyset$. Then the $\Sigma^{\wedge}$-term $t$ is $\Sigma_{0}$-defined wrt. $\left(\Sigma^{\wedge}, \mathcal{R}\right)$ iff the $\Sigma_{*}^{\wedge}$-equation $t=*$ is an induclive theorem wrt. $\left(\Sigma_{*}^{\wedge}, \mathcal{R} \cup \mathcal{R}^{*}\right)$.

Thus we can use the methods just developed to prove $\Sigma_{0}$-definedness. Let us consider again example 7.1. We have $\mathcal{R}^{*}=\{0=*, s(x)=*, p(x)=*\}$. It is easy to show that the equations $x+y=*, x-y=*$ and $x * y=*$ are inductive theorems wrt. $\left(\Sigma_{*}^{\wedge}, \mathcal{R} \cup \mathcal{R}^{*}\right)$. Then of course all $\Sigma^{\wedge}$-terms are $\Sigma_{0}$-defined wrt. $\mathcal{R}$. So we can fill the gap in the argumentation concerning example 7.1 .

To funish we briefly comment on the relationship between constructor based and unstructured specifications. Of course, the unstructured case is totally covered by our approach, as we always can decide to consider all symbols as constructors. The next result shows that, even if we are only interested in non-constructor results, it may be useful to switch over to constructor semantics, provided we have suflicient completeness.

Definition 7.1 The constructor based specification $\left(\Sigma^{\wedge}, \mathcal{R}\right)$ is said to be sufficiently complete iff every $\Sigma^{\wedge}$-term $t$ is $\Sigma_{0}$-defined wrt. $\left(\Sigma^{\wedge}, R\right)$.

Lemma 7.2 Let $\left(\Sigma^{\wedge}, \mathcal{R}\right)$ be a sufficiently complete constructor based specification induced by $\Sigma$. Then:

(a) If $\mathcal{R}$ is $\Sigma^{\wedge}$-ground confluent, then $\mathcal{R}$ is also $\Sigma$-ground confluent.

(b) If $s=t$ is an inductive theorem wrt. $\left(\Sigma^{\wedge}, \mathcal{R}\right)$, then $s=t$ is also an inductive theorem wrt. $(\Sigma, \mathcal{R})$.

Note that using this lemma we obtain "full" ground confluence in example 7.1. In an additional example (taken from [Go87] and also discussed in [Fr86]) we show that it may be convenient to switch over to the constructor based approach as one may gain efficiency.

Example 7.3 Lel $\mathcal{R}=\{R 1, R 2, R 3\}$.

$$
\begin{aligned}
& \text { (R1) } 0+y=y \\
& \text { (R2) } s(x)+y=s(x+y) \\
& \text { (R3) } x+(y+z)=(x+y)+z
\end{aligned}
$$


To show that $\mathcal{R}$ is $\Sigma$-ground confuent (in the non-constructor approach) we have to consider the following crilical pairs $C 1, \ldots, C 5$.

$$
\begin{aligned}
& \text { (C1) }(x+0)+z=x+z \\
& \text { (C2) }(0+y)+z=y+z \\
& \text { (C3) } x+s(y+z)=(x+s(y))+z \\
& \text { (C4) } s(x+(y+z))=(s(x)+y)+z \\
& \text { (C5) } x+((y+u)+v)=(x+y)+(u+v)
\end{aligned}
$$

In the constructor approach however (with the usual signature split) we only have to consider $C 1$ and $C 3$. As the specification is sufficiently complete, it suffices to prove $\Sigma^{\wedge}$-ground confluence of $C 1$ and $C 3$.

Acknowledgements: I would like to thank J. Avenhaus for some useful hints and for many discussions on earlier drafts of the paper.

\section{References}

[AvBe92] J. Avenhaus and K. Becker, Conditional rewriting modulo a built-in algebra, SEKI Report SR-92-11.

[AvMa90] J. Avenhaus and K. Madlener, Term rewriting and equational reasoning, in: R. B. Banerji, ed., Formal Techniques in Artificial Intelligence (North-Holland, Amsterdam, 1990) pp. 1-43.

[Ba88] L. Bachmair, Proof by Consistency in Equational Theories, in: 3rd LICS (1988) pp. 228-233.

[BoMo79] R.S. Boyer and J.S. Moore, A Computalional Logic (Academic Press, New York, 1979).

[Bu69] R. Burstall, Proving properties of programs by structural induction, Compuler Journal 12 (1969) pp. 41-48.

[DeJo90] N. Dershowitz and J. P. Jouannaud, Rewriting systems, in: J. van Leeuwen, ed., Handbook of Theoretical Computer Science, Vol. B (Elsevier, Ansterdam, 1990) pp. 241-320.

[Fr86] L. Fribourg, A strong restriction of the inductive completion procedure, 13th ICALP 86, LNCS 266 (Springer, Berlin, 1991) pp. 105-115.

[Go87] R. Göbel, Ground confluence, in: 2nd RTA 87 LNCS 256 (Springer, Berlin, 1991) pp. 156-167.

[HuHu82] G. Huet and J. M. Hullot, Proofs by Induction in Equational Theories with Constructors, J. Comput. Syst. Sci 25 (1982) pp. 239-266.

[KaCh86] S. Kaplan and M. Choquer, On the decidability of quasi-reducability, in: Bull. EATCS 28 (1986) pp. 32-34. 
[KaMu86] D. Kapur and D.R. Musser, Inductive reasoning for incomplete specifications, in: Proc. IEEE Symposium on Logic in Computer Science (Cambridge MA, 1986) pp. 367-377.

[KaMu87] D. Kapur and D.R. Musser, Proof by consistency, Artificial Intelligence 31(2) (1987) pp. 125-157.

[KNZ87] D. Kapur, P. Narendran and H. Zhang, On sufficient completeness and related properties of term rewriting systems, Acta Informatica 24(4) (1987), pp. 395-415.

[KNZ91] D. Kapur, P. Narendran and Il. Zlang, Automating inductionless induction using test sets, J. Symbolic Computation 11 (1991), pp. 83-111.

[KoRu90] E. Kounalis and M. Rusinowitch, Mechanizing Inductive Reasoning, in: Proc. of 8th $A A A I$ '90 (MIT Press, 1990) pp. 240-245.

[Ly92] O. Lysne, Proof by consistency in constructive systems with final algebra semantics, in: Proc. of 3rd ALP '92, LNCS 632, (Springer, Berlin 1992) pp. 276-290.

[Mu80] D. R. Musser, On Proving Inductive Properties of Abstract Data Types, in: Proc. 7th ACM Symp. on Principles of Programming Languages (1980) pp. 154-162.

[NRS89] W. Nutt, P. Réty and G. Smolka, Basic narrowing revisited, Journal of Symbolic Computation 7(3\& 4) 1989, pp. 295-318.

[Pa92] P. Padawitz, Generic induction proofs, to appear in CTRS 92.

[PI85] D. Plaisted, Semantic confluence tests and completion methods, Inform. and Control 65(2/3) 1985, pp. 182-215.

[SNGM89] G. Smolka, W. Nutt, J.A. Goguen and J. Meseguer, Order-sorted equational computation, in: H. Ait-Kaci and M. Nivat, eds., Resolution of Equations in Algebraic Struclures, Vol. 2 (Academic Press, San Diego CA, 1989) pp. 297-367.

[Wi91] C.-P. Wirth, Inductive theorem proving in theories specified by positive/negative conditional equations, Diplomarbeit, Universität Kaiserslautern, Fachbereich Informatik, 1991. 